\title{
Divulgación
}

\section{Endocarditis infecciosa por Aggregatibacter actinomycetemcomitans en un paciente con síndrome de inmunodeficiencia adquirida}

RECIBIDO: 16/04/2017 REVISIÓN: 19/07/2017

ACEPTADO: 23/08/2017

\section{Gómez Colussi, A.F.ab - Crespo, A.G. ${ }^{a}$ Mendosa, M.A. ${ }^{\text {bc }}$ - Nagel, A. ${ }^{c} \cdot$ Gutiérrez, C.E. ${ }^{\mathrm{ab}} \cdot$ Argaraña, M.F. ${ }^{\mathrm{ab}}$}

a. Hospital J.B Iturraspe, Blvd. Pellegrini 3551, Santa Fe, Santa Fe, Argentina, 3000, Tel: 0342-4575757

b. Facultad de Bioquímica y Ciencias Biológicas. Universidad Nacional del Litoral. Ciudad Universitaria UNL Ruta 168 Km 472, Santa Fe, Santa Fe, Argentina, 3000, Tel: 0342-4575216

c. Hospital J.M Cullen. Av. Freyre 2150, Santa Fe, Santa Fe, Argentina, 3000 Tel: 0342-4573340

Autor responsable de la correspondencia: Gómez Colussi Andrea andreafgc@hotmail.com

RESUMEN: Aggregatibacter

actinomycetemcomitans es una bacteria gram negativa que se encuentra formando parte de la microbiota normal de la cavidad oral en el hombre. Este microorganismo puede ocasionar cuadros infecciosos, entre ellos endocarditis. La endocarditis infecciosa (EI) es una enfermedad poco frecuente y grave, en particular si el agente causal es un microorganismo exigente, cuya identificación puede retrasarse. El diagnóstico precoz de la enfermedad, la identificación temprana de los pacientes con más riesgo, así como un abordaje multidisciplinario pueden ser estrategias válidas para mejorar el pronóstico de estos enfermos. Reportamos el primer caso en Santa Fe de El en un paciente con inmunodeficiencia adquirida (VIH), sin antecedentes de cardiopatía. El tratamiento con ceftriaxona resultó eficiente y el paciente no requirió intervención quirúrgica.

PALABRAS CLAVES: Aggregatibacter actinomycetemcomitans, periodontitis, infección invasiva, HACEK.

SUMMARY: Infective endocarditis caused by Aggregatibacter actinomycetemcomitans in a patient with acquired immunodeficiency syndrome.

Aggregatibacter actinomycetemcomitans is a gram-negative bacterium that colonizes the human oral cavity. This microorganism can cause infection, including endocarditis. 
Infective endocarditis is an unusual and serious disease, particularly if the causative agent is a fastidious microorganism whose identification could be delayed. Early diagnosis of the disease, early identification of patients at greater risk, as well as a multidisciplinary approach may be valid strategies to improve the prognosis of these patients. We report the first case in Santa Fe of infective endocarditis in a patient with acquired immunodeficiency syndrome $(\mathrm{HIV})$, without antecedents of cardiopathy. Treatment with ceftriaxone was efficient and the patient did not require surgical intervention.

KEYWORDS: Aggregatibacter actinomycetemcomitans, periodontitis, invasive infections, HACEK.

\section{Introducción}

Aggregatibacter actinomycetemcomitans es parte de la microbiota normal de la cavidad oral en individuos sanos $(1,2)$. Es el principal agente etiológico de formas agresivas de periodontitis $(3,4,5)$.

Aggregatibacter actinomycetemcomitans pertenece al grupo de microorganismos exigentes del grupo-HACEK (Haemophilus, Aggregatibacter, Cardiobacterium, Eikenella, Kingella) (6). Puede actuar como patógeno oportunista y, a partir de una enfermedad periodontal, diseminarse por vía hematógena o por secreciones a diversos órganos, produciendo endocarditis, glomerulonefritis, neumonía, osteomielitis o abscesos $(6,7,8,9,10)$. Estudios previos indican que los microorganismos del grupo HACEK son responsables del 6\% de los casos de El en el país (11).

Desde que fue aislado por primera vez en 1912 tuvo varias denominaciones, Bacterium actinomycetemcomitans (Klinger, 1912), Bacterium comitans (Lieske, 1921) y Actinobacillus actinomycetemcomitans (Topley y Wilson, 1929). Finalmente en 2006, a través de similitudes con otras especies por estudios de ADN se conformó en un nuevo género, Aggregatibacter, perteneciente a la familia Pasteurellaceae (12).
Este microorganismo es un cocobacilo gram negativo, inmóvil, capnofílico. Produce un material amorfo extracelular proteico que le permite adherirse a las células del huésped. Posee fimbrias, lipopolisacárido (LPS), leucotoxina $A$, toxina distensora del citoesqueleto (Cdt) y proteínas de adhesión e invasión celular (Omp100 y EmaA) (13).

La El es una enfermedad inflamatoria, exudativa y proliferativa del endocardio, que afecta más frecuentemente a las válvulas, y es producida por una gran variedad de microorganismos. La lesión característica es la formación de vegetaciones compuestas por plaquetas, fibrina, microorganismos y células inflamatorias que, en la mayoría de los casos, se originan a partir de pequeñas lesiones del endotelio causadas por alteraciones hemodinámicas o presencia de material extraño intracavitario.

El diagnóstico de El se basa en hallazgos clínicos, bacteriológicos y ecocardiográficos, fundamentalmente siguiendo los criterios de Duke $(14,15)$.

\section{A propósito de un caso.}

Se trata de un paciente del sexo masculino, de 55 años de edad sin hábitos de consumo de alcohol ni drogas intravenosas, con diagnóstico de infección por VIH 
que ingresó al Servicio de Clínica Médica del Hospital J.B. Iturraspe de la ciudad de Santa Fe por un cuadro febril prolongado de 20 días de evolución. La radiografía de tórax impresionó con un índice cardiotorácico aumentado con hilios engrosados, sin infiltrados evidentes y senos costodiafragmáticos libres. Se le realizaron exámenes de laboratorio que incluyeron: recuento de CD4, velocidad de sedimentación globular, hepatograma, hemograma, estado acido base, urea, creatinina. Se observó un recuento de CD4 de $189 \mathrm{cel} / \mu \mathrm{l}$, moderada leucocitosis, aumento del valor de sedimentación globular (96 mm), en tanto que la función renal, hepática y respiratoria se mostraron conservadas. Desde el punto de vista microbiológico se le efectuó la extracción de una serie de hemocultivos (dos frascos), los cuales se ingresaron en el equipo automatizado de monitoreo continuo BacT/ ALERT®. Luego de 96 h, los hemocultivos resultaron positivos. Se realizó coloración de Gram y en el examen microscópico no se observaron microorganismos. Se realizaron subcultivos en agar chocolate que fueron incubados a $37^{\circ} \mathrm{C}$ en atmósfera con $5 \%$ de $\mathrm{CO}_{2}$. Los subcultivos mostraron, luego de $48 \mathrm{~h}$, el desarrollo de colonias pequeñas, traslúcidas, de bordes irregulares y superficie rugosa fuertemente adheridas al agar. En la coloración de Gram de las colonias desarrolladas se observaron cocobacilos gram negativos pequeños. Se realizó prueba de catalasa, que resultó positiva y prueba de oxidasa con resultado negativo. En el equipo automatizado para identificación microbiana Vitek ${ }^{\circledR} 2 \mathrm{C}$ no se obtuvieron resultados concluyentes y se envió la cepa al Servicio de Bacteriología Especial INEIANLIS "Dr Carlos G. Malbrán" para identificación aplicando espectrometría de masas
(MALDI-TOF MS, Brukers, BD). El microorganismo aislado fue identificado como Aggregatibacter actinomycetemcomitans.

Ante este hallazgo, se realizaron ecocardiogramas transesofágico y transtorácico y se evidenció una imagen compatible con una vegetación sobre la cara dorsal de la valva anterior de la válvula mitral. Se instauró tratamiento con ceftriaxona intravenosa (2 g/día). El paciente mostró una evolución favorable a los 14 días de antibioticoterapia, por lo que solicitó el alta voluntaria y completó cuatro semanas de tratamiento en modalidad hospital de día.

Se describe el primer caso de endocarditis de válvula nativa por Aggregatibacter actinomycetemcomitans documentado en la ciudad de Santa Fe. Los cultivos de sangre positivos para cualquiera de los microorganismos del grupo-HACEK (Haemophilus, Aggregatibacter, Cardiobacterium, EikeneIla, Kingella) en el contexto de un foco definido de infección son altamente sugestivos de El y, al ser complementados con estudios por imágenes, contribuyen al diagnóstico definitivo del paciente. Se trata de un caso extremadamente infrecuente, ya que la asociación entre infección con VIH y El es una eventualidad a considerar en usuarios de drogas intravenosas y se destacan otros agentes etiológicos (16). Por otra parte, el estado periodontal y de la mucosa oral del paciente fue bueno y no se pudo asociar el cuadro de El con enfermedad periodontal previa. La consulta temprana del paciente podría incidir en la buena evolución del cuadro clínico. Así mismo, la implementación de sistemas de cultivo de sangre continuamente monitorizados (BacT/ALERT ${ }^{\circledR}$ ) que utilizan medios enriquecidos y de métodos de identificación rápida de microorganismos (MALDI-TOF MS), aceleran los tiempos 
de detección de las bacteriemias $(17,18)$. Con la utilización conjunta se logra un diagnóstico precoz, un tratamiento adecuado y un aumento de la sobrevida del paciente.

\section{Agradecimientos}

A la Dra. Mónica Prieto, Dra. Florencia Rocca y a los integrantes del Servicio de Bacteriología Especial INEI-ANLIS "Dr. Carlos Malbrán" de la Ciudad Autonoma de Buenos Aires por su desinteresada colaboración en la identificación del aislamiento.

\section{Referencias bibliográficas}

1. Fine, D. H.; Markowitz, K.; Furgang, D.; Velliyagounder, K. 2010. Aggregatibacter actinomycetemcomitans as an early colonizer of oral tissues: epithelium as a reservoir? J Clin Microbiol. 48, 12: 4464-4473.

2. Dewhirst, F. E.; Chen, T.; Izard, J.; Paster, B.J.; Tanner, A.C.; Yu, W.H.. 2010. The human oral microbiome. J Bacteriol. 192, 19: 5002-5017

3. Li, Y.; Messina, C.; Bendaoud, M.; Fine, D. H.; Schreiner, H.; Tsiagbe, V. K. 2010. Adaptive immune response in osteoclastic bone resorption induced by orally administered Aggregatibacter actinomycetemcomitans in a rat model of periodontal disease. Mol Oral Microbiol. 25, 4: 275-292.

4. Cortelli, J. R.; Roman-Torres, C. V. G.; Aquino, D. R.; Franco, G. C. N.; Costa, F. O.; Cortelli, S. C. 2010. Occurrence of Aggregatibacter actinomycetemcomitans in Brazilians with chronic periodontitis. Braz Oral Res. 24, 2: 217-223.

5. Ortega, S.; Vasek, O.; Sin, C. 2016. Aggregatibacter (Actinobacillus) Actinomycetemcomitans y Enfermedad Periodontal. Rev Fac Odontol. 4, 11: 29-36.

6. Yew, H. S.; Chambers, S. T.; Roberts, S. A.; Holland, D. J.; Julian, K. A.; Raymond, N. J.; Murdoch, D. R. 2014. Association between HACEK bacteraemia and endocarditis. J Med Microbiol. 63, 6: 892-895.
7. Wang, C. Y.; Wang, H. C.; Li, J. M.; Wang, J. Y.; Yang, K. C.; Ho, Y. K.; Hsueh, P. R. 2010. Invasive infections of Aggregatibacter (Actinobacillus) actinomycetemcomitans. J Microbiol Immunol Infect. 43, 6: 491-497.

8. Rahamat-Langendoen, J. C.; van Vonderen, M. G.; Engström, L. J.; Manson, W. L.; Van Winkelhoff, A. J.; Mooi-Kokenberg, E. A. 2011. Brain abscess associated with Aggregatibacter actinomycetemcomitans: case report and review of literature. J Clin Periodontol. 38, 8: 702-706.

9. Fonseca Reyes, S. 2013. Periodontitis crónica: ¿un factor de riesgo cardiovascular. Med Int Mex. 29, 5: 495-503.

10. Antony, B.; Thomas, S.; Chandrashekar, S. C.; Kumar, M. S.; Kumar, V. 2009. Osteomyelitis of the mandible due to Aggregatibacter (Actinobacillus) actinomycetemcomitans. Indian J Pathol Microbiol. 52, 1: 115.

11. Ferreiros, E.; Nacinovich, F.; Casabé, J. H.; Modenesi, J. C.; Swieszkowski, S.; Cortes, C. 2006. Epidemiologic, clinical, and microbiologic profile of infective endocarditis in Argentina: A national survey. The Endocarditis Infecciosa en la República Argentina-2 (EIRA-2) Study. Am Heart J. 151, 2: 545-552.

12. Nørskov-Lauritsen, N.; Kilian, M. 2006. Reclassification of Actinobacillus actinomycetemcomitans, Haemophilus aphrophilus, Haemophilus paraphrophilus and Haemophilus segnis as Aggregatibacter actinomycetemcomitans gen. nov., comb. nov., Aggregatibacter aphrophilus comb. nov. and Aggregatibacter segnis comb. nov., and emended description of Aggregatibacter aphrophilus to include $V$ factor-dependent and $\mathrm{V}$ factor-independent isolates. Int $\mathrm{J}$ Syst Evol Microbiol. 56, 9: 2135-2146.

13. Díaz Zúñiga, J.; Yáñez Figueroa, J.; Melgar Rodríguez, S.; Álvarez Rivas, C.; Rojas Lagos, C.; \& Vernal Astudillo, R. 2012. Virulencia y variabilidad de Porphyromonas gingivalis y Aggregatibacter actinomycetemcomitans y su asociación 
a la periodontitis. Rev Clin Periodoncia Implantol Rehabil Oral. 5,1: 40-45.

14. Mestres, C. A.; Paré, J. C.; Miró, J. M. 2015. Organización y funcionamiento de un grupo multidisciplinario de diagnóstico y tratamiento de la endocarditis infecciosa: perspectiva de 30 años (1985-2014). Rev Esp Cardiol. 68, 5: 363-368. 15. Kuster, F. 2016. Guías Europeas 2015 de endocarditis infecciosa: Nuevos desafíos, nuevas esperanzas. Rev Urug de Cardiol.31, 2: 256-260. 16. Hermida Ameijeiras, Á.; López Rodríguez, R.; Rodríguez Framil, M.;Lado Lado, F. 2007. Estudio retrospectivo de la endocarditis infecciosa en diferentes grupos de riesgo. Rev Med Chile.135, 1: 11-16.

17. García, P.; Allende, F.; Legarraga, P.; Huilcaman, M.; Solari, S. 2012. Identificación bacteriana basada en el espectro de masas de proteínas: Una nueva mirada a la microbiología del siglo XXI. Rev Chilena Infectol. 29, 3: 263-272.

18. Ferrera, C.; Vilacosta, I.; Fernández, C.; López, J.; Olmos, C.; Sarriá, C.; San Román, J. A. 2012. Reevaluación de la endocarditis con hemocultivos negativos: su perfil es similar al de la endocarditis con hemocultivos positivos. Rev Esp Cardiol. 65,10: 891-900. 